\title{
Entanglement distillation by adiabatic passage in coupled quantum dots
}

\author{
Jaroslav Fabian ${ }^{1,2}$ and Ulrich Hohenester ${ }^{1}$ \\ ${ }^{1}$ Institute of Physics, Karl-Franzens University, Universitätsplatz, 5, 8010 Graz, Austria \\ ${ }^{2}$ Institute for Theoretical Physics, University of Regensburg, 93040 Regensburg, Germany
}

(Received 9 December 2004; revised manuscript received 19 September 2005; published 18 November 2005)

\begin{abstract}
The adiabatic passage of two correlated electrons in three coupled quantum dots is shown to provide a robust and controlled way of distilling, transporting, and detecting spin entanglement, as well as measuring the rate of spin disentanglement. By employing tunable interdot coupling the scheme creates from an unentangled twoelectron state, a superposition of spatially separated singlet and triplet states. A single measurement of a dot population (charge) collapses the wave function to either of these states, realizing the entanglement to the charge conversion. The scheme is robust, with the efficiency close to $100 \%$, for a large range of realistic spectral parameters.
\end{abstract}

DOI: 10.1103/PhysRevB.72.201304

PACS number(s): 73.63.Kv, 03.67.Mn, 03.67.Hk, 03.67.Lx

The creation and detection of spin entanglement is a major task for quantum information processing. ${ }^{1}$ A particular implementation of the processing relies on electron spins in coupled quantum dots, proposed as qubits for quantum inverters $^{2}$ and for universal gating in quantum computation. ${ }^{3}$ It has been proposed that entangled two-electron spin states in quantum dots can be produced by tuned quantum gates, ${ }^{3-5}$ by correlated tunneling through single dots, ${ }^{6}$ filtering through time-dependent barriers, ${ }^{7}$ or by projective measurements. ${ }^{8,9}$ The entanglement is proposed to be detected by current noise measurements. ${ }^{10}$ Impressive recent progress in coherent control of electronic states in quantum $\operatorname{dots}^{11-14}$ and spin coherence ${ }^{15}$ gives strong impetus to these concepts. Concurrent with our proposal, several papers have appeared in the literature that discuss entanglement generation in coupled quantum dots. ${ }^{16,17}$

A typical scheme of entanglement generation relies on the Einstein-Podolsky-Rosen concept of separating two electrons from a correlated (usually singlet) ground state (see Refs. 18 and 19 for an application in quantum dots). Here we present a philosophy: instead of spatially separating individual electrons, we propose to separate correlated electron states. In our scheme the whole two-electron state goes to one side if it is a singlet and to the other side if it is a triplet, forming a spatially separated superposition of two entangled states. We propose a unique assignment of the entanglement states to charge, because by a single measurement of the charge, say, on the left, we know with almost certainty that the detected state will be a singlet. If no charge is detected, we know the state is a triplet which sits on the right. The charge measurement is nondemolition for the two states, enabling entanglement distillation. The remarkable feature of the scheme is that, unlike previous proposals, it is also capable of entanglement detection, transport, as well as disentanglement measurement, all in a robust way, without the need for fine tuning or precise knowledge of spectral or pulse parameters. We call the scheme, which can be realized by current experimental techniques, entanglement distillation by adiabatic passage (EDAP).

We model the physics of two electrons in three coupled dots (Fig. 1) by the time-dependent Hubbard Hamiltonian

$$
H=\sum_{i \lambda} \varepsilon_{i} n_{i \lambda}+\sum_{i<j, \lambda \lambda^{\prime}} U_{i \lambda, j \lambda^{\prime}} n_{i \lambda} n_{j \lambda^{\prime}}+\sum_{i j, \lambda} t_{i j} a_{i \lambda}^{\dagger} a_{j \lambda}
$$

with the Fermi creation $\left(a_{i \lambda}^{\dagger}\right)$ and annihilation $\left(a_{i \lambda}\right)$ operators for dot $i(1,2$, and 3$)$, and spin $\lambda=\uparrow, \downarrow$, and number operators $n_{i \lambda}=a_{i \lambda}^{\dagger} a_{i \lambda}$. The confining energies $\varepsilon_{i}$ do not depend on spin. We take $\varepsilon_{1}=\varepsilon_{3}=0$, while setting an offset for the middle dot $\varepsilon_{2}=\Delta$. The offset can be controlled electrostatically, or it can be fixed within a useful spectral range as shown below. We take the on-site Coulomb repulsion $U_{i \uparrow, i \downarrow}$ $=U$ to be the same for all dots, similarly for the off-site interactions $U_{i, \lambda ; i+1, \lambda^{\prime}}=V$, and zero otherwise. Hopping integrals representing interdot couplings are $t_{i j}$. For our system only $t_{12}$ and $t_{23}$ are not zero and depend on time $t$, so that $H=H(t)$. The interdot couplings are modulated by electrostatic gates defining interdot barriers. The spectral scales are meVs, with $t \ll U$ for realistic systems. In the examples below we use generic values of $U=1 \mathrm{meV}, V=0$ or $0.1 \mathrm{meV}$, and maximum hoppings smaller than $0.1 \mathrm{meV}$. Precise values will not be relevant. The Hubbard Hamiltonian does not include the higher order single-dot triplet states, whose energy can be a fraction of an meV above $U$. Inclusion of these states (one can show that they do not change the stated efficiency of our scheme for a reasonable range of parameters) would make the discussion much less transparent. In the following we present numerical results of the dynamics based

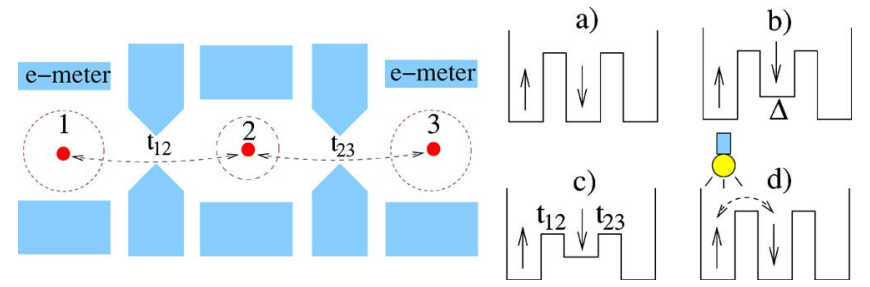

FIG. 1. (Color online) The entanglement distillation by adiabatic passage. Three quantum dots are coupled via electrode-defined barriers giving tunnel couplings $t_{12}$ and $t_{23}$. The ground state energy of dot 2 is shifted by $\Delta$. The charge on dots 1 or 3 is detected by electrometers. On the right-hand side the four figures show the scheme at work [the light bulb in (d) is an electrometer]. 


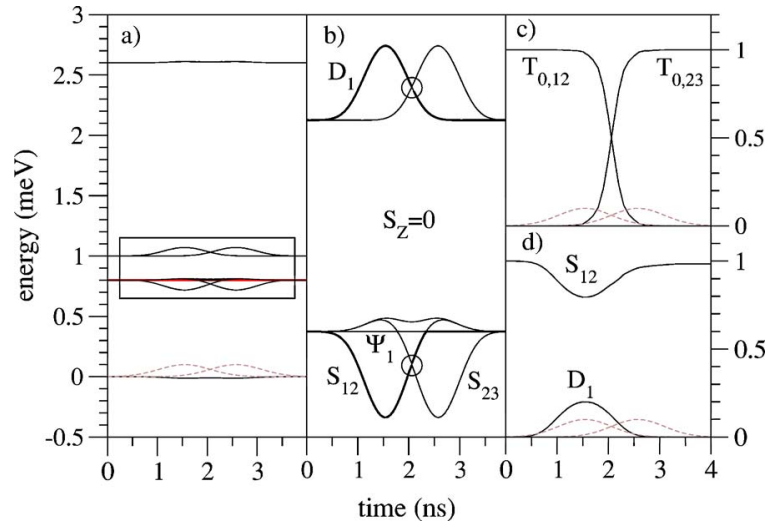

FIG. 2. (Color online) (a) Temporal evolution of the twoelectron spectrum (solid lines) of the Hamiltonian $H$ in the presence of two overlapping Gaussian pulses (dashed) of $t_{12}$ and $t_{23}$. The spectra are plotted for $U=1 \mathrm{meV}, V=0$, and $\Delta=0.8 \mathrm{meV}$. The pulses of $t_{12}(t)$ and $t_{23}(t)$ have widths $\tau \approx 500$ ps. (b) States with $S_{z}=0$ relevant for the EDAP, from the box in (a). There is a level repulsion (anticrossing) inside the circles, where the passage is rapid. At the other two crossings there is no repulsion. The horizontal line is the trapped state $\Psi_{1}$. (c) The counterintuitive passage scheme for $\Psi_{1}$ showing the probabilities $p$ of finding states $\left|T_{0}\right\rangle_{12}$ and $\left|T_{0}\right\rangle_{23}$. (d) The passage scheme for $|S\rangle_{12}$ showing the probabilities $p$ of observing $|S\rangle_{12}$ and $\left|D_{1}\right\rangle$.

on Hamiltonian (1); and later explain the results qualitatively using an analytical model of adiabatic and rapid passage using perturbation theory.

The time dependent spectrum of $H$, in the presence of interdot coupling pulses, is shown in Fig. 2(a). We take Gaussian pulses of the form $t_{i j}(t)=t_{0} \exp \left(-t^{2} / 2 \tau^{2}\right)$, where $t_{0}$ is the maximum pulse strength and $\tau$ is the dispersion. The overlap between the pulses is taken to be $2 \tau$, the width of one pulse. There are three weakly coupled groups of states. The lowest states with energy $E \approx 0$ consist of electrons occupying mainly dots 1 and 3 . The highest state, $E \approx U+2 \Delta$, is for a double occupancy of dot 2 . The states relevant for the EDAP have $E \approx U, \Delta$, and comprise electron singlets and triplets on neighboring dots. These states are magnified in Fig. 2(b). To simplify the notation we introduce the following labels for triplet $T$ and singlet $S$ states on dots $i$ and $j$ (assuming $i<j$ ), as well as for double occupancy states $D$ :

$$
\begin{gathered}
\left|T_{1}\right\rangle_{i j}=a_{i \uparrow}^{\dagger} a_{j \uparrow}^{\dagger}|0\rangle, \quad\left|T_{-1}\right\rangle_{i j}=a_{i \downarrow}^{\dagger} a_{j \downarrow}^{\dagger}|0\rangle, \\
\left|T_{0}\right\rangle_{i j}=(1 / \sqrt{2})\left(a_{i \uparrow}^{\dagger} a_{j \downarrow}^{\dagger}-a_{j \uparrow}^{\dagger} a_{i \downarrow}^{\dagger}\right)|0\rangle, \\
|S\rangle_{i j}=(1 / \sqrt{2})\left(a_{i \uparrow}^{\dagger} a_{j \downarrow}^{\dagger}+a_{j \uparrow}^{\dagger} a_{i \downarrow}^{\dagger}\right)|0\rangle, \\
|D\rangle_{i}=a_{i \uparrow}^{\dagger} a_{i \downarrow}^{\dagger}|0\rangle .
\end{gathered}
$$

Here $|0\rangle$ is the vacuum. The triplet states $\left|T_{S_{z}}\right\rangle$ are labeled by their spin $S_{z}$. States $\left|T_{0}\right\rangle$ and $|S\rangle$ are spin entangled.

We first summarize the EDAP steps and then discuss the physics in detail. The scheme is shown in Fig. 1: (a) Start with two uncoupled electrons occupying neighboring dots 1 and 2. (b) Raise slowly the energy of the middle dot 2 to $\Delta$ being on the scale of $U$ (this step is not necessary if $\Delta$ is built in). (c) Apply an overlapping pulse sequence of $t_{12}$ and $t_{23}$ (order not relevant). After the pulses fade away, $\Delta$ can be switched back to zero, if necessary. The resulting state is with a high probability, a superposition of a singlet state, spread over dots 1 and 2, and triplet states, on dots 2 and 3 . A detection of (the absence of) charge in dot 1, collapses the wave function to the singlet (triplet). Mathematically, an initial two-electron state $\Psi(t=0)$ localized on dots 1 and 2 is a superposition

$$
\Psi(0)=a|S\rangle_{12}+b\left|T_{0}\right\rangle_{12}+c\left|T_{1}\right\rangle_{12}+d\left|T_{-1}\right\rangle_{12} .
$$

After the EDAP, the state will be

$$
\Psi(\infty)=a^{\prime}|S\rangle_{12}+b^{\prime}\left|T_{0}\right\rangle_{23}+c^{\prime}\left|T_{1}\right\rangle_{23}+d^{\prime}\left|T_{-1}\right\rangle_{23},
$$

where the primed coefficients are equal to unprimed up to a phase factor. The singlet state returns to the initial dots while the triplets are transported to dots 2 and 3. As a result, the entanglement is coupled to charge on dots 1 and 3 . The scheme also works as a noninvasive entanglement detector. If the initial state is a singlet, the final state is the same (up to a phase). If it is a triplet, the state is shifted in space. Charge measurement on dots 1 or 3 , which is a nondemolition measurement for singlet and triplet states in the absence of interdot coupling, separates the two. In general, probabilities of finding, say the singlet in a given initial state, $|a|^{2}$ can be obtained by repeating the measurement on the identically prepared state, detecting a degree of entanglement. The scheme does not, however, discern the individual triplet states $\left|T_{0}\right\rangle$ and $\left|T_{ \pm 1}\right\rangle$ without an additional single-dot control (e.g., spin rotation). Finally, the scheme detects disentanglement (entanglement loss) and charge decoherence by observing systematic deviations from the expected final states (e.g., detecting charge on both 1 and 3).

To demonstrate the scheme we study the evolution of each of the states in the superposition of Eq. (6). Consider triplet states first. It is useful to find the eigenstates of $H$ whose energies do not depend on $t_{12}$ or $t_{23}$; in analogy with quantum optics, we call these states trapped. There are four twoelectron trapped states of $H$ :

$$
\begin{gathered}
\Psi_{1}=\sin \varphi\left|T_{0}\right\rangle_{12}-\cos \varphi\left|T_{0}\right\rangle_{23}, \\
\Psi_{2}=\sin \varphi\left|T_{1}\right\rangle_{12}-\cos \varphi\left|T_{1}\right\rangle_{23}, \\
\Psi_{3}=\sin \varphi\left|T_{-1}\right\rangle_{12}-\cos \varphi\left|T_{-1}\right\rangle_{23}, \\
\Psi_{4}=\left[|D\rangle_{1}-|D\rangle_{2}+|D\rangle_{3}\right] / \sqrt{3}
\end{gathered}
$$

The mixing angle $\varphi=\varphi(t)$ is defined by $\tan \varphi=t_{12} / t_{23}$. States $\Psi_{1}$ through $\Psi_{3}$ have energy $V+\Delta$, while $\Psi_{4}$, which is trapped only for $\Delta=0$, has energy $U$. As in the stimulated Raman adiabatic passage (STIRAP), which is a technique for population transfer via trapped states, ${ }^{20}$ states $\Psi_{1}$ through $\Psi_{3}$ allow the passage of an initial triplet state $|T\rangle_{12}$ to $|T\rangle_{23}$, or vice versa. Take $\Psi_{1}$ as an example. If the initial state is $\left|T_{0}\right\rangle_{12}$, it will be $100 \%$ in $\Psi_{1}$ for $t_{23}=0$, when $t_{12}$ is slowly turned on $(\varphi=\pi / 2)$. The state is unaltered until a subsequent overlapping pulse of $t_{23}$ will smoothly move the state to $\Psi_{1}=\left|T_{0}\right\rangle_{23}$, after $t_{12}$ vanishes $(\varphi=0)$. During the passage, no 
state other than the two triplets is populated. The numerical calculation is shown in Fig. 2(c), confirming the qualitative picture. In our context this pulse sequence $\left(t_{12}\right.$ before $\left.t_{23}\right)$ can be called counterintuitive, while the opposite order $\left(t_{23}\right.$ before $t_{12}$ ) intuitive. Transfer through $\Psi_{1}$ by counterintuitive sequence is extremely robust, independent on spectral parameters, as long as adiabatic conditions, to be specified, hold. While adiabatic passage via $\Psi_{1}$ is a nine-level process (there are nine $S_{z}=0$ basis states), the scheme with $\Psi_{2}$ and $\Psi_{3}$, for transporting spin unentangled triplets $\left|T_{1}\right\rangle$ and $\left|T_{-1}\right\rangle$, is an exact analog of the three level STIRAP. Triplet states can also be transferred through intuitive sequencing (not via $\Psi_{1}$ ), if $\Delta$ is greater than the interdot couplings. Such a transfer is less robust, but for our scheme it is equally satisfactory as counterintuitive, since we need $\Delta \geqslant t_{12}, t_{23}$ to transfer singlet states, as shown below. The fourth trapped state, $\Psi_{4}$, is a superposition of doubly occupied states. Because it cannot be manipulated with interdot couplings, we call this state globally trapped. We will not use this state below.

Singlet states are not part of the trapped states. If the initial state is the singlet $|S\rangle_{12}$, the above scheme in general leads to an arbitrary superposition of eigenstates of $H$ for isolated dots. There is, however, a window of energy offsets $\Delta$ where the final state will be $|S\rangle_{12}$, up to a phase. Consider states $|S\rangle_{12},|D\rangle_{1}$, and $|D\rangle_{2}$ with average energies $\Delta+V, U$, and $2 \Delta+U$, respectively. If we make $\Delta$ on the same scale as $U$, state $|D\rangle_{2}$, as well as all other eigenstates, will not be easily accessible due to spectral separation [Fig. 2(a)]. We have an effective two-level system with Hamiltonian (up to a constant)

$$
H^{\prime}=\frac{1}{2}(\Delta+V-U) \sigma_{z}+\sqrt{2} t_{12}(t) \sigma_{x},
$$

where $\sigma_{\alpha}$ are the Pauli matrices. The eigenstates are

$$
\begin{aligned}
& \Psi_{+}=\cos (\vartheta / 2)|S\rangle_{12}+\sin (\vartheta / 2)|D\rangle_{1}, \\
& \Psi_{-}=\sin (\vartheta / 2)|S\rangle_{12}-\cos (\vartheta / 2)|D\rangle_{1} .
\end{aligned}
$$

The mixing angle $\vartheta=\vartheta(t)$, restricted to $[0, \pi]$, is defined by $\tan \vartheta=2 \sqrt{2} t_{12} /(\Delta+V-U)$. The nature of the time evolution of the singlet depends critically on $\Delta$. In resonance, $\Delta+V$ $\approx U$, the singlet is initially a superposition of $\Psi_{+}$and $\Psi_{-}$. After passage of pulse $t_{12}$ the final state will be $\Psi(\infty)$ $=|S\rangle_{12} \cos \alpha+|D\rangle_{1} \sin \alpha$, where the pulse area $\alpha$ $=\int \sqrt{2} t_{12}(t) d t$. By fine tuning the pulses to $\alpha=\pi$, the final state will be $|S\rangle_{12}$.

The above resonant scheme for singles, though allowing fine control, is not robust: it requires both the resonance condition and precise knowledge of the pulse area. We instead explore the large spectral window off the resonance. For $|\Delta+V-U| \geqslant t_{12}$, state $|S\rangle_{12}$ will be transported back to itself, via $\Psi_{+}$. (This is analogous to adiabatically following a spin along a magnetic field that rotates along the $y$ axis back and forth adiabatically.) Such a passage is very robust. The twolevel picture is confirmed by the numerical calculation with the full Hamiltonian $H$ in Fig. 2(d).

Figure 3 shows the EDAP results as a function of $\Delta$, for two initial states. For the selected $\tau$ the initial singlet returns to the same state at least $90 \%$ of times for $\Delta \geq 0.6 \mathrm{meV}$

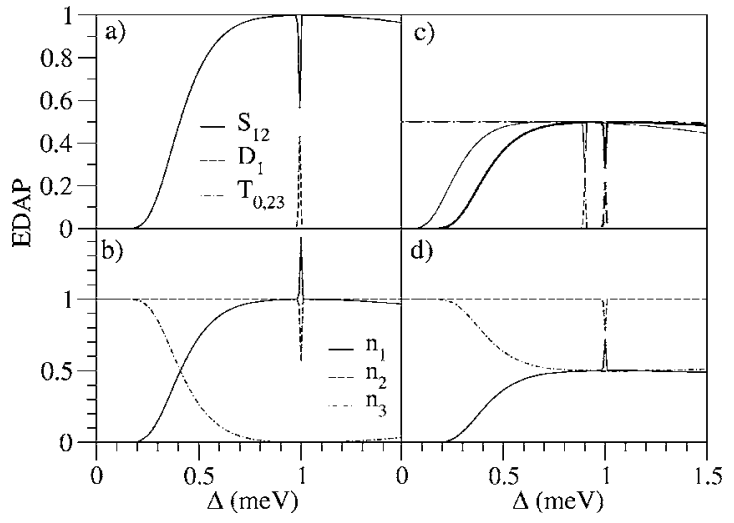

FIG. 3. (a) The calculated probabilities and electron populations after the EDAP as a function of $\Delta$, with $\Psi(0)=|S\rangle_{12}[$ (a) and (b)] and $\Psi(0)=a_{1 \uparrow}^{\dagger} a_{2 \downarrow}^{\dagger}|0\rangle[(\mathrm{c})$ and $(\mathrm{d})]$. The thin dotted lines in (c) are for $V=0.1 \mathrm{meV}$. The pulses are the same as in Fig. 2 .

(unless at resonance visible by spikes). This is closely mirrored by the charge population of the dots 1 and 3. Dot 2 has always charge one, except for resonance, in which dot 1 can be doubly occupied. The influence of off-site Coulomb interaction is seen in Fig. 3(c). The only effect is shifting the resonance from $\Delta=U$ to $\Delta=U-V$.

What is the condition on the pulse? Passage of state $|l\rangle$ is adiabatic if $|\langle l|\hbar d H(t) / d t| k\rangle| \ll\left(\hbar \omega_{l k}\right)^{2}$, where $k$ are other eigenstates of $H(t)$, and $\omega_{l k}$ are the Bohr frequencies. ${ }^{21} \mathrm{We}$ give rough estimates for the limits on pulse dispersion $\tau$ (switching time), based on the qualitative criterion that the smallest relevant Bohr period needs to be resolved during the passage. The EDAP comprises four processes: (i) Adiabatic passage of the triplet state. For $|\Delta| \leq t_{0}$, which can be used for triplet transport, this is robust if $\tau \gtrsim \hbar / t_{0}$. In our scheme $\Delta \gg t_{0}$ and the smallest relevant Bohr energy is $t_{0}^{2} / \Delta$. Then $\tau \gtrsim \tau_{L}$ where $\tau_{L}=\hbar^{2} /\left(t_{0}^{2} / \tau\right)$ gives the lower limit. (ii) Adiabatic passage of the singlet. This is a two-level scheme with states separated by $\sim t_{0}$. Thus $\tau \gtrsim \hbar / t_{0}$, which is within the range of (ii) and need not be considered extra. (iii) Rapid passage of the singlet through the anticrossing at time $t$ given by $\bar{t}=t_{12}(t)=t_{23}(t)$ [Fig. 2(b)]. The level repulsion is small, since $|S\rangle_{12}$ couples to $|S\rangle_{23}$ through spectrally distant states such as $|S\rangle_{13}$ and $|D\rangle_{2}$. Interference in the virtual coupling to these states further reduces the anticrossing. One can show that the level spacing is $V_{g} \approx 2\left(\vec{t}^{2} / \Delta\right)(U-\Delta) /(U+\Delta)$, vanishingly small at resonance in the order $O\left(\vec{t}^{2} / \Delta\right)$. Rapid passage occurs for $\tau^{\prime} \lesssim \hbar / V_{g}$, where $\tau^{\prime}=\tau V_{g} / t_{0}$ is the time over which the interdot coupling changes by $V_{g}$, relevant for resolving the gap. This gives $\tau \lesssim \tau_{U}$ where $\tau_{U}=\tau_{L} \times\left(\Delta / t_{0}\right)(U$ $+\Delta) /(U-\Delta)$ is the upper limit. Finally, (iv) the EDAP has to be performed within the coherence time of the system, which is, at low temperatures, likely in the nanosecond time scale. ${ }^{13,14}$ Considering full coherence, the time limitations on the pulse are $\tau_{L} \lesssim \tau \lesssim \tau_{U}$, which for our model parameters is $100 \mathrm{ps}$ to $10 \mathrm{~ns}$. Since the lower limit is given by energy $t_{0}^{2} / \Delta$ which is on the order of the exchange coupling ( $J$ $=t_{0}^{2} / U$ in the Hubbard model) for our case of $\Delta \approx U$, the times are similar to those used for spin-based quantum computing. ${ }^{4}$ The upper limit $\tau_{U}$ increases with decreasing 
$|U-\Delta|$. The scheme will perform quadratically faster for larger couplings.

To identify numerically the regime of applicability of the scheme, we define the EDAP efficiency $w$ as

$$
w=\left|\langle\Psi(\infty) \mid S\rangle_{12}\right|^{2}+\left|\left\langle\Psi(\infty) \mid T_{0}\right\rangle_{23}\right|^{2},
$$

for a state $\Psi(t)$ with the initial condition $\Psi(0)=a_{1 \uparrow}^{\dagger} a_{2 \downarrow}^{\dagger}|0\rangle$. This definition is insensitive to the relative phase change, and to the relative population of the two states. The efficiency is plotted in Fig. 4 as a function of $\Delta$ and $\tau$ for the counterintuitive sequence (intuitive shows the same picture except at $\Delta$ close to 0 ). The range of applicability, from $100 \mathrm{ps}$ to $10 \mathrm{~ns}$ agrees with our analytical estimates for our parameters. The graph also shows the predicted increase of applicable $\tau$ with decreasing $|U-\Delta|$. It is evident that our scheme is very robust, covering a large range of spectral values and pulse times. The horizontal "cut" at $\Delta=U$ $=1 \mathrm{meV}$ indicates the resonance oscillations of the $|S\rangle_{12}$ $-|D\rangle_{1}$ pair for which the efficiency depends on the area of the pulse, $\alpha$, and thus on $\tau$. The lower limit on $\tau$ can be further reduced by about a decade (to $50 \mathrm{ps}$ for $98 \%$ efficiency for our parameters) by decreasing the delay between the pulses (not shown here).

Efficiency $w$ can be measured by performing the EDAP twice: if the first (distillation) passage results in, say, a singlet, the second (detection) passage should give absence of charge on dot 3 , if $w \approx 1$. Another interesting application of the EDAP can be in quantifying the influence of a charge probe on the charge itself. Say, use the EDAP to transport triplets via $\Psi_{1}$. Since $n_{2} \Psi_{1}=\Psi_{1}$ at all times [in fact, $\Psi_{1}$

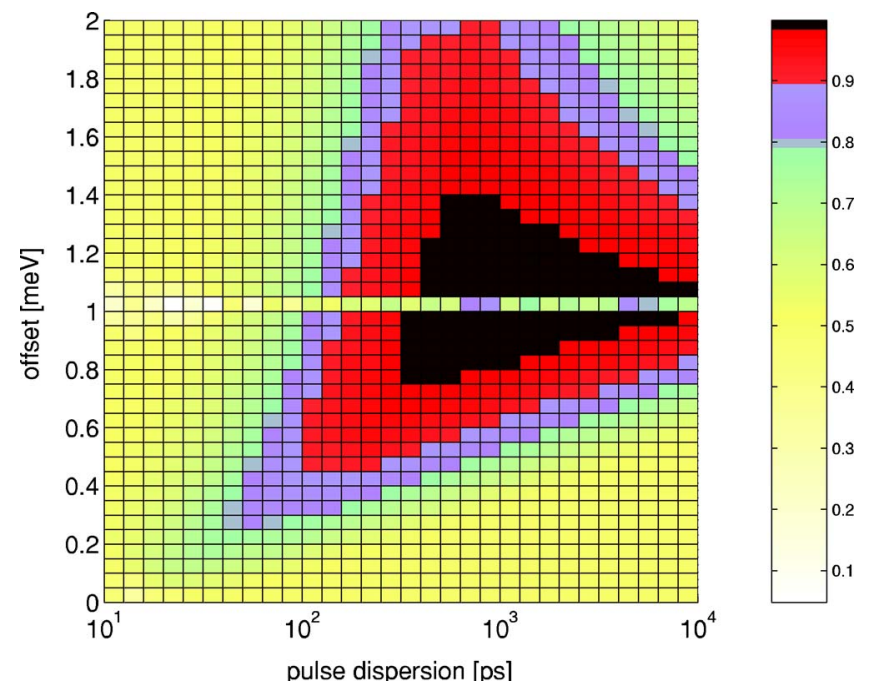

FIG. 4. (Color online) The calculated efficiency $w$ as a function of the pulse dispersion $\tau$ and offset $\Delta$, for the counterintuitive pulse sequence. The black (darkest) window is for efficiency higher than $98 \%$, while the second darkest window is for $w>90 \%$.

through $\Psi_{3}$ are the only eigenstates of $H(t)$ that are also eigenstates of $n_{2}$ ], a measurement of population on dot 2 should not disturb the state. The EDAP efficiency loss is a measure of the invasiveness of the probe.

We thank Peter Zoller for useful discussions. This work was supported by the U.S. ONR and FWF.
${ }^{1}$ C. H. Bennett and D. P. DiVincenzo, Nature (London) 404, 247 (2000).

${ }^{2}$ S. Bandyopadhyay and V. P. Roychowdhury, Superlattices Microstruct. 22, 411 (1997).

${ }^{3}$ D. Loss and D. P. DiVincenzo, Phys. Rev. A 57, 120 (1998).

${ }^{4}$ G. Burkard, D. Loss, and D. P. DiVincenzo, Phys. Rev. B 59, 2070 (1999).

${ }^{5}$ X. Hu and S. Das Sarma, Phys. Rev. A 61, 062301 (2000).

${ }^{6}$ W. D. Oliver, F. Yamaguchi, and Y. Yamamoto, Phys. Rev. Lett. 88, 037901 (2002).

${ }^{7}$ X. Hu and S. Das Sarma, Phys. Rev. B 69, 115312 (2004).

${ }^{8}$ R. Ruskov and A. N. Korotkov, Phys. Rev. B 67, 241305(R) (2003).

${ }^{9}$ T. M. Stace, S. D. Barrett, H. S. Goan, and G. J. Milburn, Phys. Rev. B 70, 205342 (2004).

${ }^{10}$ D. Loss and E. V. Sukhorukov, Phys. Rev. Lett. 84, 1035 (2000).

${ }^{11}$ W. G. van der Wiel, S. De Franceschi, J. M. Elzerman, T. Fujisawa, S. Tarucha, and L. P. Kouwenhoven, Rev. Mod. Phys. 75, 1 (2003).

${ }^{12}$ T. Hayashi, T. Fujisawa, H. D. Cheong, Y. H. Jeong, and Y.
Hirayama, Phys. Rev. Lett. 91, 226804 (2003).

${ }^{13}$ J. R. Petta, A. C. Johnson, C. M. Marcus, M. P. Hanson, and A. C. Gossard, Phys. Rev. Lett. 93, 186802 (2004).

${ }^{14}$ J. R. Petta, A. C. Johnson, A. Jacoby, C. M. Marcus, M. P. Hanson, and A. C. Gossard, cond-mat/0412048 (unpublished).

${ }^{15}$ R. Hanson, B. Witkamp, L. M. K. Vandersypen, L. H. Willems van Beveren, J. M. Elzerman, and L. P. Kouwenhoven, Phys. Rev. Lett. 91, 196802 (2003).

${ }^{16}$ J. M. Taylor, W. Dur, P. Zoller, A. Yacoby, C. M. Marcus, and M. D. Lukin, Phys. Rev. Lett. 94, 236803 (2005).

${ }^{17}$ M. Blaauboer and D. DiVincenzo, Phys. Rev. Lett. 95, 160402 (2005).

${ }^{18}$ D. S. Saraga and D. Loss, Phys. Rev. Lett. 90, 166803 (2003).

${ }^{19}$ P. Zhang, Q. K. Xue, X. G. Zhao, and X. C. Xie, Phys. Rev. A 69, 042307 (2004).

${ }^{20}$ K. Bergmann, H. Theuer, and B. W. Shore, Rev. Mod. Phys. 70, 1003 (1998).

${ }^{21}$ A. Messiah, Quantum Mechanics (North-Holland, Amsterdam, 1965), Vol. II. 